JOURNAL OF THE

AMERICAN MATHEMATICAL SOCIETY

Volume 23, Number 2, April 2010, Pages 469-490

S 0894-0347(09)00651-1

Article electronically published on November 13, 2009

\title{
EXISTENCE OF MINIMAL MODELS FOR VARIETIES OF LOG GENERAL TYPE II
}

\author{
CHRISTOPHER D. HACON AND JAMES M ${ }^{\mathrm{C}}$ KERNAN
}

\section{Contents}

1. Introduction 469

2. Notation and conventions 473

3. Preliminary results 475

4. Multiplier ideal sheaves 477

5. Asymptotic multiplier ideal sheaves 4

6. Lifting sections 485

7. Rationality of the restricted algebra 487

8. Proof of Theorem $1.3 \quad 488$

References

\section{INTRODUCTION}

This is the second of two papers whose purpose is to establish:

Theorem 1.1. The canonical ring

$$
R\left(X, K_{X}\right)=\bigoplus_{m \in \mathbb{N}} H^{0}\left(X, \mathcal{O}_{X}\left(m K_{X}\right)\right)
$$

is finitely generated for every smooth projective variety $X$.

Note that Siu has announced a proof of finite generation for varieties of general type, using analytic methods; see [13.

Our proof relies on the ideas and techniques of the minimal model program and roughly speaking in this paper we will show that finite generation in dimension $n-1$ implies the existence of flips in dimension $n$. To be more precise, we first recall some definitions:

Received by the editors August 13, 2008.

2010 Mathematics Subject Classification. Primary 14E30.

The first author was partially supported by NSF research grant no. 0456363 and an AMS Centennial fellowship.

The second author was partially supported by NSA grant no. H98230-06-1-0059 and NSF grant no. 0701101 .

The authors would like to thank F. Ambro, C. Birkar, P. Cascini, J. A. Chen, A. Corti, O. Fujino, S. Keel, J. Kollár and the referee for valuable suggestions.

(C)2009 American Mathematical Society Reverts to public domain 28 years from publication 
Definition 1.2. Let $\pi: X \longrightarrow U$ be a projective morphism of normal quasiprojective varieties, and let $V$ be a finite dimensional affine subspace of the real vector $\operatorname{space}_{\mathrm{WDiv}_{\mathbb{R}}}(X)$ of Weil divisors on $X$. Fix an $\mathbb{R}$-divisor $A \geq 0$ and define

$$
\begin{aligned}
V_{A} & =\{\Delta \mid \Delta=A+B, B \in V\}, \\
\mathcal{L}_{A}(V) & =\left\{\Delta=A+B \in V_{A} \mid K_{X}+\Delta \text { is log canonical and } B \geq 0\right\} .
\end{aligned}
$$

Then, assuming the following:

Theorem F. Let $\pi: X \longrightarrow Z$ be a projective morphism to a normal affine variety. Let $(X, \Delta=A+B)$ be a $\mathbb{Q}$-factorial Kawamata log terminal pair of dimension $n$, where $A \geq 0$ is an ample $\mathbb{Q}$-divisor and $B \geq 0$. If $K_{X}+\Delta$ is pseudo-effective, then

(1) The pair $(X, \Delta)$ has a log terminal model $\mu: X \rightarrow Y$. In particular if $K_{X}+\Delta$ is $\mathbb{Q}$-Cartier, then the log canonical ring

$$
R\left(X, K_{X}+\Delta\right)=\bigoplus_{m \in \mathbb{N}} H^{0}\left(X, \mathcal{O}_{X}\left(\left\llcorner m\left(K_{X}+\Delta\right)\right\lrcorner\right)\right),
$$

is finitely generated.

(2) Let $V \subset \operatorname{WDiv}_{\mathbb{R}}(X)$ be the vector space spanned by the components of $\Delta$. Then there is a constant $\delta>0$ such that if $G$ is a prime divisor contained in the stable base locus of $K_{X}+\Delta$ and $\Xi \in \mathcal{L}_{A}(V)$ such that $\|\Xi-\Delta\|<\delta$, then $G$ is contained in the stable base locus of $K_{X}+\Xi$.

(3) Let $W \subset V$ be the smallest affine subspace of $\operatorname{WDiv}_{\mathbb{R}}(X)$ containing $\Delta$, which is defined over the rationals. Then there is a constant $\eta>0$ and a positive integer $r>0$ such that if $\Xi \in W$ is any divisor and $k$ is any positive integer such that $\|\Xi-\Delta\|<\eta$ and $k\left(K_{X}+\Xi\right) / r$ is Cartier, then every component of $\operatorname{Fix}\left(k\left(K_{X}+\Xi\right)\right)$ is a component of the stable base locus of $K_{X}+\Delta$.

we prove the existence of pl-flips:

Theorem A. Pl-flips exist in dimension $n$.

Theorem $\mathrm{F}$ and Theorem $\mathrm{A}$ are taken directly from 2 . Theorem $\mathrm{A}$, refers to Theorem $\mathrm{A}$ in the case when the dimension of $X$ is $n$.

Theorem 1.3. Theorem $\mathrm{F}_{h-1}$ implies Theorem $\mathrm{A}_{h}$.

It is proved in 2 that Theorem 1.3 completes the proof of Theorem 1.1 .

The main ideas used in this paper have their origins in the work of Shokurov on the existence of flips [12] together with the use of the extension theorem of [5], which in turn was inspired by the work of Kawamata, Siu and Tsuji (cf. 77, 14] and [17). For further history about the details of this problem, see [3, §2.1].

In this paper, however, we do not make use of the concept of "asymptotic saturation" introduced by Shokurov, and in fact we prove a more general result which does not require the relative weak log Fano condition (see also [1]).

Further treatments of the results of this paper may be found in [1] and [6] (which follows Shokurov's approach more explicitly).

We now turn to a more detailed description of the results and techniques used in this paper. Recall the following:

Definition 1.4. Let $(X, \Delta)$ be a purely $\log$ terminal pair and $f: X \longrightarrow Z$ be a projective morphism of normal varieties. Then $f$ is a pl-flipping contraction if $\Delta$ is a $\mathbb{Q}$-divisor and 
(1) $f$ is small, of relative Picard number one,

(2) $-\left(K_{X}+\Delta\right)$ is $f$-ample,

(3) $X$ is $\mathbb{Q}$-factorial,

(4) $S=\llcorner\Delta\lrcorner$ is irreducible and $-S$ is $f$-ample.

The flip of a pl-flipping contraction $f: X \longrightarrow Z$ is a small projective morphism $g: Y \longrightarrow Z$ of relative Picard number one, such that $K_{Y}+\Gamma$ is $g$-ample, where $\Gamma$ is the strict transform of $\Delta$.

The flip $g$ is unique, if it exists at all, and it is given by

$$
Y=\operatorname{Proj}_{Z} \mathfrak{R}, \quad \text { where } \quad \mathfrak{R}=\bigoplus_{m \in \mathbb{N}: k \mid m} f_{*} \mathcal{O}_{X}\left(m\left(K_{X}+\Delta\right)\right),
$$

and $k$ is any positive integer such that $k\left(K_{X}+\Delta\right)$ is integral. Therefore, in order to prove the existence of pl-flips, it suffices to show that $\mathfrak{R}$ is a finitely generated $\mathcal{O}_{Z}$-algebra. Since this problem is local over $Z$, we may assume that $Z=\operatorname{Spec} A$ is affine and it suffices to prove that

$$
R\left(X, k\left(K_{X}+\Delta\right)\right)=\bigoplus_{m \in \mathbb{N}: k \mid m} H^{0}\left(X, \mathcal{O}_{X}\left(m\left(K_{X}+\Delta\right)\right)\right)
$$

is a finitely generated $A$-algebra. It is then natural to consider the restricted algebra

$$
R_{S}\left(X, k\left(K_{X}+\Delta\right)\right)=\operatorname{Im}\left(R\left(X, k\left(K_{X}+\Delta\right)\right) \longrightarrow R\left(S, k\left(K_{S}+\Omega\right)\right)\right),
$$

whose graded pieces correspond to the images of the restriction homomorphisms

$$
H^{0}\left(X, \mathcal{O}_{X}\left(m\left(K_{X}+\Delta\right)\right)\right) \longrightarrow H^{0}\left(S, \mathcal{O}_{S}\left(m\left(K_{S}+\Omega\right)\right)\right),
$$

where $m=k l$ is divisible by $k$ and $\Omega$ is defined by the adjunction formula

$$
\left.\left(K_{X}+\Delta\right)\right|_{S}=K_{S}+\Omega,
$$

and $k\left(K_{X}+\Delta\right)$ is Cartier. Shokurov has shown, cf. Theorem 3.2, that the algebra $R\left(X, k\left(K_{X}+\Delta\right)\right)$ is finitely generated if and only if the restricted algebra is finitely generated.

Now, if the natural inclusion

$$
R_{S}\left(X, k\left(K_{X}+\Delta\right)\right) \subset R\left(S, k\left(K_{S}+\Omega\right)\right)
$$

were an isomorphism, then Theorem 1.3 would follow from (1) of Theorem $\mathrm{F}_{h-1}$. In fact the pair $(S, \Omega)$ is Kawamata $\log$ terminal, $\operatorname{dim} S=\operatorname{dim} X-1=n-1$ and since $\left.f\right|_{S}$ is birational, $\Omega$ is automatically big so that, by a standard argument, (1) of Theorem $\mathrm{F}_{-1}$ applies and $R\left(S, k\left(K_{S}+\Omega\right)\right)$ is finitely generated. Theorem 3.2 also implies that $\mathfrak{R}$ is finitely generated.

Unluckily this is too much to hope for. However it does suggest that one should concentrate on the problem of lifting sections, and the main focus of this paper is to prove the extension result, Theorem 6.3. In fact, Theorem 1.3 is a straightforward consequence of Theorem 6.3 .

To fix ideas, let us start with an example where we cannot lift sections. Let $X$ be the blowup of $\mathbb{P}^{2}$ at a point $o$, with exceptional divisor $E$. Let $S$ be the strict transform of a line through $o$, let $L_{1}, L_{2}$ and $L_{3}$ be the strict transforms of general lines in $\mathbb{P}^{2}$, let $p=E \cap S$ and let $p_{i}=L_{i} \cap S$. Then the pair

$$
\left(X, \Delta=S+(2 / 3)\left(E+L_{1}+L_{2}+L_{3}\right)\right)
$$


is purely log terminal, but the homomorphism

$$
H^{0}\left(\mathcal{O}_{X}\left(3 l\left(K_{X}+\Delta\right)\right)\right) \longrightarrow H^{0}\left(\mathcal{O}_{S}\left(3 l\left(K_{S}+\Omega\right)\right)\right) \simeq H^{0}\left(\mathcal{O}_{\mathbb{P}^{1}}(2 l)\right)
$$

is never surjective, where $\Omega=\left.(\Delta-S)\right|_{S}=(2 / 3)\left(p+p_{1}+p_{2}+p_{3}\right)$ and $l$ is a positive integer. The problem is that the stable base locus of $K_{X}+\Delta$ contains $E$ and yet $\left|3\left(K_{S}+\Omega\right)\right|$ is base point free. Notice, however, that

$$
\left|3 l\left(K_{X}+\Delta\right)\right|_{S}=\left|3 l\left(K_{S}+\Theta\right)\right|+3 l(\Omega-\Theta),
$$

where $\Theta=(2 / 3)\left(p_{1}+p_{2}+p_{3}\right)$ is obtained from $\Omega$ by throwing away $p$. In other words, $\Theta$ is obtained from $\Omega$ by removing some part of each component contained in the stable base locus of $K_{X}+\Delta$.

Returning to the general setting, one may then hope that the restricted algebra $R_{S}\left(S, l\left(K_{X}+\Delta\right)\right)$ is given by an algebra of the form $R\left(S, l\left(K_{S}+\Theta\right)\right)$ for some Kawamata $\log$ terminal pair $(S, \Theta)$, where $0 \leq \Theta \leq \Omega$ is a $\mathbb{Q}$-divisor obtained from $\Omega$ by subtracting components of $\Omega$ contained in the stable base locus of $K_{X}+\Delta$. We will now explain how this may be achieved. The tricky thing is to determine exactly how much of the stable base locus to throw away.

It is not hard to reduce to the following situation: $\pi: X \longrightarrow Z$ is a projective morphism to a normal affine variety $Z$, where $(X, \Delta=S+A+B)$ is a purely log terminal pair of dimension $n, S=\llcorner\Delta\lrcorner$ is irreducible, $X$ and $S$ are smooth, $A \geq 0$ is an ample $\mathbb{Q}$-divisor, $B \geq 0,\left(S, \Omega=\left.(\Delta-S)\right|_{S}\right)$ is canonical and the stable base locus of $K_{X}+\Delta$ does not contain $S$.

Let

$$
\Theta_{m}=\Omega-\Omega \wedge F_{m}, \quad \text { where } \quad F_{m}=\operatorname{Fix}\left(\left|m\left(K_{X}+\Delta\right)\right|_{S}\right) / m,
$$

and $m\left(K_{X}+\Delta\right)$ is Cartier. $\Omega \wedge F_{m}$ is the minimum of $\Omega$ and $F_{m}$, where the minimum is taken component by component. In particular $m\left(\Omega-\Theta_{m}\right)$ is the biggest divisor contained in $\operatorname{Fix}\left(\left|m\left(K_{X}+\Delta\right)\right|_{S}\right)$ such that $0 \leq \Theta_{m} \leq \Omega$. It follows that

$$
\left|m\left(K_{S}+\Theta_{m}\right)\right|+m\left(\Omega-\Theta_{m}\right) \supset\left|m\left(K_{X}+\Delta\right)\right|_{S} .
$$

A simple consequence of the main lifting result, Theorem 6.3. of this paper implies that this tautological inclusion $(\mathbb{D})$ is actually an equality,

$$
\left|m\left(K_{S}+\Theta_{m}\right)\right|+m\left(\Omega-\Theta_{m}\right)=\left|m\left(K_{X}+\Delta\right)\right|_{S} .
$$

A technical, but significant, improvement on the proof of the existence of flips which appears in [6] is that the statement of $(\equiv)$ and of Theorem 6.3 involves only linear systems and divisors on $X$ and $S$, even though the proof of Theorem 6.3 involves passing to a higher model. The key point is that since $(S, \Omega)$ is canonical, it suffices to keep track only of the fixed divisor on $S$ and not of the whole base locus.

To prove (国) we use the method of multiplier ideal sheaves. In fact the main point is to establish an inclusion of multiplier ideal sheaves, Theorem 5.3. A proof of Theorem 5.3 appeared originally in $[5$. We chose to include a proof of this result for the convenience of the reader and we decided to use notation closer to the wellestablished notation used in [10. Note however that the multiplier ideal sheaves we use, see Definition-Lemma 4.2, must take into account the divisor $\Delta$ (for example consider the case worked out above) and the fact that $(S, \Omega)$ is canonical.

In fact, if one assumes the MMP, then one should expect (国) to hold. Indeed, if one runs $f: X \rightarrow Y$ the $\left(K_{X}+\Delta\right)$-MMP, almost by definition this will not change the linear systems $\left|m\left(K_{X}+\Delta\right)\right|$. Since $K_{Y}+\Gamma=K_{X}+f_{*} \Delta$ is nef, one can lift 
sections on $Y$ from the strict transform $T$ of $S$, by an easy application of KawamataViehweg vanishing. In general, however, the linear systems $\left|m\left(K_{T}+g_{*} \Theta\right)\right|$ are bigger than the linear systems $\left|m\left(K_{S}+\Theta\right)\right|$, since the induced birational map $g: S \rightarrow T$ might extract some divisors. However any such divisor must have log discrepancy at most one, so this cannot happen, almost by definition, if $K_{S}+\Theta$ is canonical.

In order to establish that $R_{S}\left(X, k\left(K_{X}+\Delta\right)\right)$ is finitely generated, cf. Theorem 7.1. and thereby to finish the proof of Theorem 1.3] it is necessary and sufficient to show that $\Theta=\lim \left(\Theta_{m !} / m !\right)$ is rational (the seemingly strange use of factorials is so that we can use limits rather than limsups). At this point we play off two facts. The first is that, since we are assuming that Theorem $\mathrm{F}$ holds on $S$, if $m>0$ is sufficiently divisible and $\Phi$ is an appropriately chosen $\mathbb{Q}$-divisor sufficiently close to $\Theta$, then the base locus of $\left|m\left(K_{S}+\Phi\right)\right|$ and the stable base locus of $K_{S}+\Theta$ are essentially the same (basically because $K_{S}+\Theta$ and $K_{S}+\Phi$ share a log terminal model $\mu: S \rightarrow S^{\prime}$ and these two sets of divisors are precisely the divisors contracted by $\mu$ ). The second is that using Theorem 4.1, Theorem 6.3 is slightly stronger than (回); one is allowed to overshoot $\Theta_{m}$ by an amount $\epsilon / m$, where $\epsilon>0$ is fixed. (It seems worth pointing out that Theorem 4.1 seems to us a little mysterious. In particular, unlike Theorem 6.3. we were unable to show that this result follows from the MMP.)

More precisely, since the base locus of $\left|m\left(K_{S}+\Theta_{m}\right)\right|$ contains no components of $\Theta_{m}$, by (2) of Theorem $\mathrm{E}$ it follows that the stable base locus of $K_{S}+\Theta$ contains no components of $\Theta$. If $\Theta$ is not rational, then by Diophantine approximation there is a $\mathbb{Q}$-divisor $0 \leq \Phi \leq \Omega$ very close to $\Theta$ and an integer $k>0$ such that $k \Phi$ is integral and $\operatorname{mult}_{G} \Phi>\operatorname{mult}_{G} \Theta$, for some prime divisor $G$. By Theorem 6.3, it actually follows that

$$
\left|k\left(K_{S}+\Phi\right)\right|+k(\Omega-\Phi)=\left|k\left(K_{X}+\Delta\right)\right|_{S} .
$$

The condition mult ${ }_{G} \Phi>\operatorname{mult}_{G} \Theta$ ensures that $G$ is a component of $\operatorname{Fix}\left(k\left(K_{S}+\Phi\right)\right)$, and hence of the stable base locus of $K_{S}+\Phi$. But then $G$ is a component of $\Theta$ and of the stable base locus of $K_{S}+\Theta$. This is the required contradiction.

\section{Notation And CONVEntions}

We work over the field of complex numbers $\mathbb{C}$. Let $X$ be a normal variety. A

$$
\left.\begin{array}{r}
\text { (integral) divisor } \\
\mathbb{Q} \text {-divisor } \\
\mathbb{R} \text {-divisor }
\end{array}\right\} \text { is a } \quad\left\{\begin{array}{c}
\mathbb{Z} \text {-linear } \\
\mathbb{Q} \text {-linear } \\
\mathbb{R} \text {-linear }
\end{array}\right.
$$

combination of prime divisors. Given an integral Weil divisor $D$, we let

$$
R(X, D)=\bigoplus_{m \in \mathbb{N}} H^{0}\left(X, \mathcal{O}_{X}(m D)\right)
$$

Set

$$
\begin{aligned}
& \operatorname{WDiv}_{\mathbb{Q}}(X)=\operatorname{WDiv}(X) \underset{\mathbb{Z}}{\otimes} \mathbb{Q}, \\
& \operatorname{WDiv}_{\mathbb{R}}(X)=\operatorname{WDiv}(X) \underset{\mathbb{Z}}{\underset{\mathbb{R}}{\otimes}} \mathbb{R},
\end{aligned}
$$

where $\operatorname{WDiv}(X)$ is the group of Weil divisors on $X$. The definitions below for $\mathbb{R}$ divisors reduce to the usual definitions for $\mathbb{Q}$-divisors and integral divisors; see [2]. Note that the group of $\mathbb{R}$-divisors forms a vector space, with a canonical basis given 
by the prime divisors. If $C=\sum c_{i} B_{i}$ and $D=\sum d_{i} B_{i}$, where the $B_{i}$ are distinct prime divisors, then we write $D \geq 0$ if $d_{i} \geq 0$ and we will denote by

$$
\begin{aligned}
\|C\| & =\max _{i} c_{i}, & C \wedge D & =\sum_{i} \min \left\{c_{i}, d_{i}\right\} B_{i}, \\
\llcorner C\lrcorner & =\sum_{i}\left\llcorner c_{i}\right\lrcorner B_{i}, & \{C\} & =C-\llcorner C\lrcorner .
\end{aligned}
$$

Two $\mathbb{R}$-divisors $C$ and $D$ are

$$
\left.\begin{array}{ll}
\text { linearly equivalent, } & C \sim D \\
\mathbb{Q} \text {-linearly equivalent, } & C \sim \mathbb{Q} D \\
\mathbb{R} \text {-linearly equivalent, } & C \sim \mathbb{R} D
\end{array}\right\} \quad \text { if } C-D \text { is a }\left\{\begin{array}{l}
\mathbb{Z} \text {-linear } \\
\mathbb{Q} \text {-linear } \\
\mathbb{R} \text {-linear }
\end{array}\right.
$$

combination of principal divisors. Note that if $C \sim_{\mathbb{Q}} D$, then $m C \sim m D$ for some positive integer $m$, but this fails in general for $\mathbb{R}$-linear equivalence. Note also that if two $\mathbb{Q}$-divisors are $\mathbb{R}$-linearly equivalent, then they are in fact $\mathbb{Q}$-linearly equivalent, but that two integral divisors might be $\mathbb{Q}$-linearly equivalent without being linearly equivalent. Let

$$
\begin{aligned}
|D| & =\{C \in \operatorname{WDiv}(X) \mid C \geq 0, C \sim D\}, \\
|D|_{\mathbb{Q}} & =\left\{C \in \operatorname{WDiv}_{\mathbb{Q}}(X) \mid C \geq 0, C \sim_{\mathbb{Q}} D\right\}, \\
|D|_{\mathbb{R}} & =\left\{C \in \operatorname{Wiv}_{\mathbb{R}}(X) \mid C \geq 0, C \sim_{\mathbb{R}} D\right\} .
\end{aligned}
$$

If $T$ is a subvariety of $X$, not contained in the base locus of $|D|$, then $|D|_{T}$ denotes the image of the linear system $|D|$ under restriction to $T$. If $D$ is an integral divisor, $\operatorname{Fix}(D)$ denotes the fixed divisor of $D$ so that $|D|=|D-\operatorname{Fix}(D)|+\operatorname{Fix}(D)$, where the base locus of $|D-\operatorname{Fix}(D)|$ contains no divisors. More generally $\operatorname{Fix}(V)$ denotes the fixed divisor of the linear system $V$.

The stable base locus of $D$, denoted by $\mathbf{B}(D)$, is the intersection of the support of the elements of $|D|_{\mathbb{R}}$ (if $|D|_{\mathbb{R}}$ is empty, then by convention the stable base locus is the whole of $X$ ). The stable fixed divisor is the divisorial support of the stable base locus. The augmented stable base locus of $D$, denoted by $\mathbf{B}_{+}(D)$, is given by the stable base locus of $D-\epsilon A$ for some ample divisor $A$ and any rational number $0<\epsilon \ll 1$. The diminished stable base locus is defined by

$$
\mathbf{B}_{-}(D)=\bigcup_{\epsilon>0} \mathbf{B}(D+\epsilon A) \text {. }
$$

In particular we have

$$
\mathbf{B}_{-}(D) \subset \mathbf{B}(D) \subset \mathbf{B}_{+}(D) .
$$

This notation was established in 4 for projective varieties, but we will use it in a slightly more general setting; see for example [11.

An $\mathbb{R}$-Cartier divisor $D$ is an $\mathbb{R}$-linear combination of Cartier divisors. An $\mathbb{R}$ Cartier divisor $D$ is nef if $D \cdot \Sigma \geq 0$ for any curve $\Sigma \subset X$. An $\mathbb{R}$-Cartier divisor $D$ is ample if it is $\mathbb{R}$-linearly equivalent to a positive linear combination of ample divisors (in the usual sense). An $\mathbb{R}$-Cartier divisor $D$ is big if $D \sim \mathbb{R} A+B$, where $A$ is ample and $B \geq 0$. A $\mathbb{Q}$-Cartier divisor $D$ is a general ample $\mathbb{Q}$-divisor if there is an integer $m>0$ such that $m D$ is very ample and $m D \in|m D|$ is very general.

A $\log$ pair $(X, \Delta)$ is a normal variety $X$ and an $\mathbb{R}$-Weil divisor $\Delta \geq 0$ such that $K_{X}+\Delta$ is $\mathbb{R}$-Cartier. We say that a log pair $(X, \Delta)$ is log smooth if $X$ is smooth and the support of $\Delta$ is a divisor with global normal crossings. A projective birational morphism $g: Y \longrightarrow X$ is a $\log$ resolution of the pair $(X, \Delta)$ if $X$ is smooth and 
the inverse image of $\Delta$ union the exceptional locus is a divisor with global normal crossings. Note that in the definition of $\log$ resolution we place no requirement that the indeterminacy locus of $g$ is contained in the locus where the pair $(X, \Delta)$ is not $\log$ smooth. If $V$ is a linear system on $X$, a $\log$ resolution of $V$ and $(X, \Delta)$ is a $\log$ resolution of the pair $(X, \Delta)$ such that if $|M|+F$ is the decomposition of $g^{*} V$ into its mobile and fixed parts, then $|M|$ is base point free and $F$ union the exceptional locus union the strict transform of $\Delta$ is a divisor with simple normal crossings support. If $g$ is a $\log$ resolution, then we may write

$$
K_{Y}+\Gamma=g^{*}\left(K_{X}+\Delta\right)+E,
$$

where $\Gamma \geq 0$ and $E \geq 0$ have no common components, $g_{*} \Gamma=\Delta$ and $E$ is $g$ exceptional. Note that this decomposition is unique. The log discrepancy of a divisor $F$ over $X$ is

$$
a(X, \Delta, F)=1+\operatorname{mult}_{F}(E-\Gamma) .
$$

Note that with this definition, a component $F$ of $\Delta$ with coefficient $b$ has log discrepancy $1-b$. The log discrepancy does not depend on the choice of model $Y$, so that the $\log$ discrepancy is also a function defined on valuations. A nonKawamata log terminal place is any valuation of log discrepancy at most zero, and the centre of a non-Kawamata log terminal place is called a non-Kawamata log terminal centre. A non-terminal centre is any centre of log discrepancy at most one. Note that every divisor on $X$ is by definition a non-terminal centre, so the only interesting non-terminal centres are of codimension at least two.

The pair $(X, \Delta)$ is Kawamata log terminal if there are no non-Kawamata log terminal centres. We say that the pair $(X, \Delta)$ is purely log terminal (respectively canonical or terminal) if the log discrepancy of any exceptional divisor is greater than zero (respectively at least one or greater than one). We say that the pair is divisorially log terminal if there is a $\log$ resolution $g: Y \longrightarrow X$ such that all exceptional divisors $E \subset Y$ have log discrepancy greater than zero.

\section{Preliminary Results}

In this section we recall several results about finitely generated algebras and in particular we will give a proof of Shokurov's result that the pl-flip exists if and only if the restricted algebra is finitely generated.

Definition 3.1. Let $X$ be a normal variety, $S$ be a prime divisor and $B$ an integral Weil divisor which is $\mathbb{Q}$-Cartier and whose support does not contain $S$. The restricted algebra $R_{S}(X, B)$ is the image of the homomorphism $R(X, B) \longrightarrow$ $R\left(S,\left.B\right|_{S}\right)$.

We remark that as $B$ is $\mathbb{Q}$-Cartier, then $\left.B\right|_{S}$ is a well-defined $\mathbb{Q}$-Cartier divisor on $S$.

Theorem 3.2. Let $f: X \longrightarrow Z$ be a pl-flipping contraction with respect to $(X, \Delta)$. Pick an integer $k$ such that $k\left(K_{X}+\Delta\right)$ is Cartier.

Then

(1) The flip of $f$ exists if and only if the flip of $f$ exists locally over $Z$.

(2) If $Z=\operatorname{Spec} A$ is affine, then the flip $f^{+}: X^{+} \longrightarrow Z$ exists if and only if the restricted algebra $R_{S}\left(X, k\left(K_{X}+\Delta\right)\right)$ is a finitely generated $A$-algebra. 
Definition 3.3. Let $A$ be a ring and let $R$ be any graded $A$-algebra. A truncation of $R$ is any $A$-algebra of the form

$$
R_{(d)}=\bigoplus_{m \in \mathbb{N}} R_{m d}
$$

for a positive integer $d$.

We start with the following well-known result:

Lemma 3.4. Let $R$ be a graded algebra which is an integral domain and let d be a positive integer.

Then $R$ is finitely generated if and only if $R_{(d)}$ is finitely generated.

Proof. Suppose that $R$ is finitely generated. It is easy to write down an action of the cyclic group $\mathbb{Z}_{d}$ on $R$ so that the invariant ring is $R_{(d)}$. Thus $R_{(d)}$ is finitely generated by the Theorem of E. Noether which states that the ring of invariants of a finitely generated ring under the action of a finite group is finitely generated.

Suppose now that $R_{(d)}$ is finitely generated. Let $f \in R_{i}$. Then $f$ is a root of the monic polynomial $x^{d}-f^{d} \in R_{(d)}[x]$. It follows that $R$ is integral over $R_{(d)}$ and the result follows by another Theorem of E. Noether on the finiteness of integral closures.

Lemma 3.5. Let $S$ be a normal prime divisor on $X$ and let $B$ be an integral Weil divisor which is $\mathbb{Q}$-Cartier and whose support does not contain $S$.

- If $R(X, B)$ is finitely generated, then $R_{S}(X, B)$ is finitely generated.

- If $S \sim B$ and $R_{S}(X, B)$ is finitely generated, then $R(X, B)$ is finitely generated.

Proof. Since there is a surjective homomorphism $\phi: R(X, B) \longrightarrow R_{S}(X, B)$, it is clear that if $R(X, B)$ is finitely generated, then $R_{S}(X, B)$ is finitely generated.

Suppose now that $R_{S}(X, B)$ is finitely generated and $S \sim B$. Then there is a rational function $g_{1}$ such that $\left(g_{1}\right)=S-B$. If we consider the elements of $R(X, B)_{m}$ as rational functions, then a rational function $g$ belongs to $R(X, B)_{m}$ if and only if $(g)+m B \geq 0$. But if $g$ is in the kernel of $\phi$, then there is a divisor $S^{\prime} \geq 0$ such that $(g)+m B=S+S^{\prime}$. It follows that $\left(g / g_{1}\right)+(m-1) B=S^{\prime}$ so that $g / g_{1}=h \in R(X, B)_{m-1}$. But then the kernel of $\phi$ is the principal ideal generated by $g_{1}$.

Proof of Theorem 3.2. It is well known that the flip $f^{+}: X^{+} \longrightarrow Z$ exists if and only if the sheaf of graded $\mathcal{O}_{Z}$-algebras

$$
\bigoplus_{m \in \mathbb{N}: k \mid m} f_{*} \mathcal{O}_{X}\left(m\left(K_{X}+\Delta\right)\right)
$$

is finitely generated; cf. $[8,6.4]$. Since this can be checked locally, this gives (1).

If $Z=\operatorname{Spec} A$ is affine it suffices to check that $R\left(X, k\left(K_{X}+\Delta\right)\right)$ is a finitely generated $A$-algebra. Since the relative Picard number is one, there are real numbers $a$ and $b$ such that $a\left(K_{X}+\Delta\right)$ and $b S$ are numerically equivalent over $Z$. As both $-\left(K_{X}+\Delta\right)$ and $-S$ are ample $\mathbb{Q}$-divisors we may assume that $a$ and $b$ are both positive integers. Moreover, as $a\left(K_{X}+\Delta\right)-b S$ is numerically trivial over $Z$, it is semiample over $Z$ by the base point free theorem. In particular, we may replace numerical equivalence by linear equivalence,

$$
a\left(K_{X}+\Delta\right) \sim_{Z} b S .
$$


But then there is a rational function $g$ and a divisor $D$ on $Z$ such that

$$
a\left(K_{X}+\Delta\right)=b S+f^{*} D+(g) .
$$

As any line bundle on a quasi-projective variety is locally trivial, possibly passing to an open subset of $Z$, and using (1), we may assume that $D \sim 0$, so that

$$
a\left(K_{X}+\Delta\right) \sim b S .
$$

By Lemma 3.4 it follows that $R\left(X, k\left(K_{X}+\Delta\right)\right)$ is finitely generated if and only if $R(X, S)$ is finitely generated. Since $Z$ is affine and $f$ is small, $S$ is mobile (that is, the fixed divisor is empty) so that $S \sim S^{\prime}$, where $S^{\prime} \geq 0$ is a divisor whose support does not contain $S$. By Lemma 3.5. $R(X, S)$ is finitely generated if and only if $R_{S}\left(X, S^{\prime}\right)$ is finitely generated. Since $\left.\left.a\left(K_{X}+\Delta\right)\right|_{S} \sim b S^{\prime}\right|_{S}$ the result follows by Lemma 3.4

\section{Multiplier ideal Sheaves}

The main result of this section is:

Theorem 4.1. Let $\pi: X \longrightarrow Z$ be a projective morphism to a normal affine variety $Z$, where $(X, \Delta=S+A+B)$ is a log pair, $S=\llcorner\Delta\lrcorner$ is irreducible, $(X, S)$ is log smooth, and both $A \geq 0$ and $B \geq 0$ are $\mathbb{Q}$-divisors. Let $k$ be any positive integer and $0 \leq \Phi \leq \Omega=\left.(\Delta-S)\right|_{S}$ be any divisor such that both $k\left(K_{S}+\Phi\right)$ and $k\left(K_{X}+\Delta\right)$ are Cartier. Let $C=A / k$.

If there is an integer $l>1$ and an integral divisor $P \geq 0$ such that $l A$ is Cartier, $C-\frac{(k-1)}{m} P$ is ample, $\left(X, \Delta+\frac{k-1}{m} P\right)$ is purely log terminal and

$$
l\left|k\left(K_{S}+\Phi\right)\right|+m(\Omega-\Phi)+\left.(m C+P)\right|_{S} \subset\left|m\left(K_{X}+\Delta+C\right)+P\right|_{S},
$$

where $m=k l$, then

$$
\left|k\left(K_{S}+\Phi\right)\right|+k(\Omega-\Phi) \subset\left|k\left(K_{X}+\Delta\right)\right|_{S} .
$$

See also [14] and [16] for related statements. To prove Theorem 4.1, we need a variant of multiplier ideal sheaves:

Definition-Lemma 4.2. Let $(X, \Delta)$ be a $\log$ smooth pair, where $\Delta$ is a reduced divisor, and let $V$ be a linear system whose base locus contains no non-Kawamata $\log$ terminal centres of $(X, \Delta)$. Let $\mu: Y \longrightarrow X$ be a $\log$ resolution of $V$ and $(X, \Delta)$ and let $F$ be the fixed divisor of the linear system $\mu^{*} V$. Let $K_{Y}+\Gamma=$ $\mu^{*}\left(K_{X}+\Delta\right)+E$, where $\Gamma=\sum P_{i}$ is the sum of the divisors on $Y$ of $\log$ discrepancy zero.

Then for any real number $c \geq 0$, define the multiplier ideal sheaf

$$
\mathcal{J}_{\Delta, c \cdot V}:=\mu_{*} \mathcal{O}_{Y}(E-\llcorner c F\lrcorner) .
$$

If $\Delta=0$ we will write $\mathcal{J}_{c \cdot V}$ and if $D=c G$, where $G>0$ is a Cartier divisor, we define

where $V=\{G\}$.

$$
\mathcal{J}_{\Delta, D}:=\mathcal{J}_{\Delta, c \cdot V},
$$

Proof. We have to show that the definition of the multiplier ideal sheaf is independent of the choice of $\log$ resolution. Let $\mu: Y \longrightarrow X$ and $\mu^{\prime}: Y^{\prime} \longrightarrow X$ be two 
$\log$ resolutions of $(X, \Delta)$ and $V$. We may assume that $\mu^{\prime}$ factors through $\mu$ via a morphism $\nu: Y^{\prime} \longrightarrow Y$. Then $F^{\prime}=\nu^{*} F$ as $\mu^{*} V-F$ is free, and

$$
\begin{aligned}
E^{\prime}-c F^{\prime} & =K_{Y^{\prime}}+\Gamma^{\prime}-\mu^{\prime *}\left(K_{X}+\Delta\right)-c F^{\prime} \\
& =K_{Y^{\prime}}+\Gamma^{\prime}-\nu^{*}\left(K_{Y}+\Gamma-E+c F\right) \\
& =\nu^{*}(E-\llcorner c F\lrcorner)+K_{Y^{\prime}}+\Gamma^{\prime}-\nu^{*}\left(K_{Y}+\Gamma+\{c F\}\right) \\
& =\nu^{*}(E-\llcorner c F\lrcorner)+G .
\end{aligned}
$$

Since $(Y, \Gamma+E+F)$ is $\log$ smooth, it follows that $(Y, \Gamma+\{c F\})$ is $\log$ canonical and has the same non-Kawamata log terminal places as $(Y, \Gamma)$ and hence as $(X, \Delta)$. Thus $\ulcorner G\urcorner \geq 0$ and since $\nu_{*}\left(K_{Y^{\prime}}+\Gamma^{\prime}\right)=K_{Y}+\Gamma,\ulcorner G\urcorner$ is $\nu$-exceptional. Then

$$
\begin{aligned}
\mu_{*}^{\prime} \mathcal{O}_{Y^{\prime}}\left(E^{\prime}-\left\llcorner c F^{\prime}\right\lrcorner\right) & =\mu_{*}\left(\nu_{*} \mathcal{O}_{Y^{\prime}}\left(E^{\prime}-\left\llcorner c F^{\prime}\right\lrcorner\right)\right) \\
& =\mu_{*}\left(\nu_{*} \mathcal{O}_{Y^{\prime}}\left(\nu^{*}(E-\llcorner c F\lrcorner)+\ulcorner G\urcorner\right)\right) \\
& =\mu_{*} \mathcal{O}_{Y}(E-\llcorner c F\lrcorner) .
\end{aligned}
$$

We need to develop a little of the theory of multiplier ideal sheaves.

Lemma 4.3. Let $(X, \Delta)$ be a log smooth pair where $\Delta$ is reduced, let $V$ be a linear system whose base locus contains no non-Kawamata log terminal centres of $(X, \Delta)$ and let $G \geq 0$ and $D \geq 0$ be $\mathbb{Q}$-Cartier divisors whose supports contain no nonKawamata log terminal centres of $(X, \Delta)$.

Then

(1) $\mathcal{J}_{\Delta, D}=\mathcal{O}_{X}$ if and only if $(X, \Delta+D)$ is divisorially log terminal and $\llcorner D\lrcorner=0$.

(2) If $0 \leq \Delta^{\prime} \leq \Delta$, then $\mathcal{J}_{\Delta, c \cdot V} \subset \mathcal{J}_{\Delta^{\prime}, c \cdot V}$. In particular, $\mathcal{J}_{\Delta, c \cdot V} \subset \mathcal{J}_{c \cdot V} \subset \mathcal{O}_{X}$.

(3) If $\Sigma \geq 0$ is a Cartier divisor, $D-\Sigma \leq G$ and $\mathcal{J}_{\Delta, G}=\mathcal{O}_{X}$, then $\mathcal{I}_{\Sigma} \subset \mathcal{J}_{\Delta, D}$.

Proof. (1) follows easily from the definitions.

(2) follows from the fact that $a\left(P, X, \Delta^{\prime}\right) \geq a(P, X, \Delta)$ for all divisors $P$ on $Y$.

To see (3), note that we are free to replace $\Sigma$ by a smaller Cartier divisor $\Sigma^{\prime}$ such that $D-\Sigma^{\prime} \leq G$. As $D$ contains no non-Kawamata log terminal centres of $(X, \Delta)$ we may therefore assume that $\Sigma$ contains no non-Kawamata log terminal centres of $(X, \Delta)$ as well. Notice that as $\Sigma$ is Cartier and $\mathcal{J}_{\Delta, G}=\mathcal{O}_{X}$, we have

$$
\mathcal{J}_{\Delta, G}(-\Sigma)=\mathcal{O}_{X}(-\Sigma)=\mathcal{I}_{\Sigma}
$$

But since $D \leq G+\Sigma$ and $\Sigma$ contains no non-Kawamata log terminal centres of $(X, \Delta)$ we also have

$$
\mathcal{J}_{\Delta, G}(-\Sigma)=\mathcal{J}_{\Delta, G+\Sigma} \subset \mathcal{J}_{\Delta, D}
$$

We have the following extension of (9.5.1) of [9] or (2.4.2) of [16]:

Lemma 4.4. Let $\pi: X \longrightarrow Z$ be a projective morphism to a normal affine variety $Z$. Let $(X, \Delta)$ be a log smooth pair where $\Delta$ is reduced, let $S$ be a component of $\Delta$, let $D \geq 0$ be a $\mathbb{Q}$-Cartier divisor whose support does not contain any non-Kawamata log terminal centres of $(X, \Delta)$ and let $\Theta=\left.(\Delta-S)\right|_{S}$. Let $N$ be a Cartier divisor.

(1) There is a short exact sequence

$$
0 \longrightarrow \mathcal{J}_{\Delta-S, D+S} \longrightarrow \mathcal{J}_{\Delta, D} \longrightarrow \mathcal{J}_{\Theta,\left.D\right|_{S}} \longrightarrow 0 .
$$


(2) (Nadel Vanishing) If $N-D$ is ample, then

$$
H^{i}\left(X, \mathcal{J}_{\Delta, D}\left(K_{X}+\Delta+N\right)\right)=0
$$

for $i>0$.

(3) If $N-D$ is ample, then

$$
H^{0}\left(X, \mathcal{J}_{\Delta, D}\left(K_{X}+\Delta+N\right)\right) \longrightarrow H^{0}\left(S, \mathcal{J}_{\Theta, D \mid S}\left(K_{X}+\Delta+N\right)\right)
$$

is surjective.

Proof. By the resolution lemma of [15], we may find a $\log$ resolution $\mu: Y \longrightarrow X$ of $(X, \Delta+D)$ which is an isomorphism over the generic point of each non-Kawamata $\log$ terminal centre of $(X, \Delta)$. If $T$ is the strict transform of $S$, then we have a short exact sequence

$$
0 \longrightarrow \mathcal{O}_{Y}\left(E-\left\llcorner\mu^{*} D\right\lrcorner-T\right) \longrightarrow \mathcal{O}_{Y}\left(E-\left\llcorner\mu^{*} D\right\lrcorner\right) \longrightarrow \mathcal{O}_{T}\left(E-\left\llcorner\mu^{*} D\right\lrcorner\right) \longrightarrow 0,
$$

where $E$ is defined in Definition-Lemma 4.2. Now $\mu_{*} \mathcal{O}_{Y}\left(E-\left\llcorner\mu^{*} D\right\lrcorner\right)=\mathcal{J}_{\Delta, D}$. If $\Gamma$ is the sum of the divisors of $\log$ discrepancy zero, then

$$
E-\mu^{*} D=\left(K_{Y}+\Gamma\right)-\mu^{*}\left(K_{X}+\Delta+D\right) .
$$

But then

$$
E-\mu^{*} D-T=\left(K_{Y}+\Gamma-T\right)-\mu^{*}\left(K_{X}+\Delta-S+(D+S)\right)
$$

so that

$$
\mu_{*} \mathcal{O}_{Y}\left(E-\left\llcorner\mu^{*} D\right\lrcorner-T\right)=\mathcal{J}_{\Delta-S, D+S}
$$

and

so that

$$
\left.\left(E-\mu^{*} D\right)\right|_{T}=K_{T}+\left.(\Gamma-T)\right|_{T}-\mu^{*}\left(K_{S}+\Theta+\left.D\right|_{S}\right),
$$

$$
\mu_{*} \mathcal{O}_{T}\left(E-\left\llcorner\mu^{*} D\right\lrcorner\right)=\mathcal{J}_{\Theta,\left.D\right|_{S}} .
$$

Since $\left(Y, \Gamma+\mu^{*} D\right)$ is $\log$ smooth and $\Gamma$ and $\mu^{*} D$ have no common components, $\left(Y, \Gamma+\left\{\mu^{*} D\right\}\right)$ is divisorially log terminal. Choose $F \geq 0$ such that $-F$ is $\mu$-ample whose coefficients are sufficiently small so that $K_{Y}+\Gamma+\left\{\mu^{*} D\right\}+F$ is divisorially $\log$ terminal. As

$$
E-\left\llcorner\mu^{*} D\right\lrcorner-T-\left(K_{Y}+\Gamma-T+\left\{\mu^{*} D\right\}+F\right)=-\mu^{*}\left(K_{X}+\Delta+D\right)-F
$$

is $\mu$-ample, Kawamata-Viehweg vanishing implies that

$$
R^{1} \mu_{*} \mathcal{O}_{Y}\left(E-\left\llcorner\mu^{*} D\right\lrcorner-T\right)=0,
$$

and this gives (1).

Similarly, Kawamata-Viehweg vanishing implies that

$$
R^{i} \mu_{*} \mathcal{O}_{Y}\left(\mu^{*}\left(K_{X}+\Delta+N\right)+E-\left\llcorner\mu^{*} D\right\lrcorner\right)=0,
$$

for $i>0$. As $N-D$ is ample, then, possibly replacing $F$ by a small multiple, we may assume that $\mu^{*}(N-D)-F$ is ample. As

$$
\mu^{*}\left(K_{X}+\Delta+N\right)+E-\left\llcorner\mu^{*} D\right\lrcorner-\left(K_{Y}+\Gamma+\left\{\mu^{*} D\right\}+F\right)=\mu^{*}(N-D)-F
$$

is ample, Kawamata-Viehweg vanishing implies that

$$
H^{i}\left(Y, \mathcal{O}_{Y}\left(\mu^{*}\left(K_{X}+\Delta+N\right)+E-\left\llcorner\mu^{*} D\right\lrcorner\right)\right)=0,
$$

for $i>0$. Since the Leray-Serre spectral sequence degenerates, this gives (2), and (3) follows from (2). 
Proof of Theorem 4.1. Since $\left(X, \Delta+\frac{k-1}{m} P\right)$ is purely log terminal, $\left(S, \Omega+\left.\frac{k-1}{m} P\right|_{S}\right)$ is Kawamata $\log$ terminal and $S$ is not contained in the support of $A$ or $P$. If $\Sigma \in\left|k\left(K_{S}+\Phi\right)\right|$, then we may pick a divisor

$$
G \in\left|m\left(K_{X}+\Delta+C\right)+P\right| \text { such that }\left.G\right|_{S}=l \Sigma+m\left(\Omega-\Phi+\left.C\right|_{S}\right)+\left.P\right|_{S} .
$$

Let

$$
\Lambda=\frac{k-1}{m} G+B \quad \text { and } \quad N=k\left(K_{X}+\Delta\right)-K_{X}-S .
$$

As the support of the $\mathbb{Q}$-divisor $\Lambda \geq 0$ does not contain $S$ and by assumption

$$
N-\Lambda \sim_{\mathbb{Q}} C-\frac{k-1}{m} P
$$

is ample, Lemma 4.4 implies that sections of $H^{0}\left(S, \mathcal{J}_{\left.\Lambda\right|_{S}}\left(k\left(K_{S}+\Omega\right)\right)\right)$ extend to sections of $H^{0}\left(X, \mathcal{O}_{X}\left(k\left(K_{X}+\Delta\right)\right)\right)$. Now

$$
\begin{aligned}
& \left.\Lambda\right|_{S}-(\Sigma+k(\Omega-\Phi)) \\
= & \frac{k-1}{m}\left(l \Sigma+m\left(\Omega-\Phi+\left.C\right|_{S}\right)+\left.P\right|_{S}\right)+\left.B\right|_{S}-(\Sigma+k(\Omega-\Phi)) \\
\leq & \Omega+\left.\frac{k-1}{m} P\right|_{S} .
\end{aligned}
$$

As $\left(S, \Omega+\left.\frac{k-1}{m} P\right|_{S}\right)$ is Kawamata $\log$ terminal, $\mathcal{J}_{\Omega+\left.\frac{k-1}{m} P\right|_{S}}=\mathcal{O}_{S}$ and we are done by (3) of Lemma 4.3 .

\section{Asymptotic multiplier ideal Sheaves}

Definition 5.1. Let $X$ be a normal variety and let $D$ be a divisor. An additive sequence of linear systems associated to $D$ is a sequence $V_{\bullet}$ such that $V_{m} \subset$ $\mathbb{P}\left(H^{0}\left(X, \mathcal{O}_{X}(m D)\right)\right)$ and

$$
V_{i}+V_{j} \subset V_{i+j}
$$

Definition-Lemma 5.2. Suppose that $(X, \Delta)$ is $\log$ smooth, where $\Delta$ is reduced and let $V_{\bullet}$ be an additive sequence of linear systems associated to a divisor $D$. Assume that there is an integer $k>0$ such that no non-Kawamata log terminal centre of $(X, \Delta)$ is contained in the base locus of $V_{k}$.

If $c$ is a positive real number and $p$ and $q$ are positive integers divisible by $k$, then

$$
\mathcal{J}_{\Delta, \frac{c}{p} \cdot V_{p}} \subset \mathcal{J}_{\Delta, \frac{c}{q} \cdot V_{q}} \quad \forall q \text { divisible by } p .
$$

In particular the asymptotic multiplier ideal sheaf of $V_{\bullet}$,

$$
\mathcal{J}_{\Delta, c \cdot V_{\bullet}}=\bigcup_{p>0} \mathcal{J}_{\Delta, \frac{c}{p} \cdot V_{p}}
$$

is given by $\mathcal{J}_{\Delta, c \cdot V \bullet}=\mathcal{J}_{\Delta, \frac{c}{p} \cdot V_{p}}$, for $p$ sufficiently large and divisible. If we take $V_{m}=|m D|$ the complete linear system, then define

$$
\mathcal{J}_{\Delta, c \cdot\|D\|}=\mathcal{J}_{\Delta, c \cdot V_{\bullet}},
$$

and if $S$ is a component of $\Delta$ and we take $W_{m}=|m D|_{S}$, then define

$$
\mathcal{J}_{\Theta, c \cdot\|D\|_{S}}=\mathcal{J}_{\Theta, c \cdot W_{\bullet}}
$$

where $\Theta=\left.(\Delta-S)\right|_{S}$. 
Proof. If $p$ divides $q$, then pick a common $\log$ resolution $\mu: Y \longrightarrow X$ of $V_{p}, V_{q}$ and $(X, \Delta)$ and note that

$$
\frac{1}{q} F_{q} \leq \frac{1}{p} F_{p}
$$

where $F_{p}$ is the fixed locus of $\mu^{*} V_{p}$ and $F_{q}$ is the fixed locus of $\mu^{*} V_{q}$. Therefore $\mathcal{J}_{\Delta, \frac{c}{p} \cdot V_{p}} \subset \mathcal{J}_{\Delta, \frac{c}{q} \cdot V_{q}}$. The equality $\mathcal{J}_{\Delta, c \cdot V_{\bullet}}=\mathcal{J}_{\Delta, \frac{c}{p} \cdot V_{p}}$ now follows as $X$ is Noetherian.

We are now ready to state the main result of this section:

Theorem 5.3. Let $\pi: X \longrightarrow Z$ be a projective morphism to a normal affine variety $Z$. Suppose that $(X, \Delta=S+B)$ is log smooth and purely log terminal of dimension $n$, where $S=\llcorner\Delta\lrcorner$ is irreducible, and let $k$ be a positive integer such that $D=$ $k\left(K_{X}+\Delta\right)$ is integral. Let $A$ be any ample $\mathbb{Q}$-divisor on $X$. Let $q$ and $r$ be any positive integers such that $Q=q A$ is very ample, $r A$ is Cartier and $(j-1) K_{X}+\Xi+$ $r A$ is ample for every Cartier divisor $0 \leq \Xi \leq j\ulcorner\Delta\urcorner$ and every integer $1 \leq j \leq k+1$.

If the stable base locus of $D$ does not contain any non-Kawamata log terminal centres of $(X,\ulcorner\Delta\urcorner)$, then

$$
\mathcal{J}_{\left\|\left.m D\right|_{S}\right\|} \subset \mathcal{J}_{\Theta,\|m D+P\|_{S}} \quad \text { for all } \quad m \in \mathbb{N},
$$

where $\Theta=\left.\ulcorner B\urcorner\right|_{S}, p=q n+r$ and $P=p A$. Moreover, all sections of the linear system determined by $\mathcal{J}_{\|m D \mid s\|}(m D+P)$ lift; that is, we have

$$
\pi_{*} \mathcal{J}_{\left\|\left.m D\right|_{S}\right\|}(m D+P) \subset \operatorname{Im}\left(\pi_{*} \mathcal{O}_{X}(m D+P) \rightarrow \pi_{*} \mathcal{O}_{S}(m D+P)\right),
$$

for all $m \in \mathbb{N}$.

We will need some results about the sheaves $\mathcal{J}_{\Delta, c \cdot V_{\bullet}}$, most of which are easy generalisations of the corresponding facts for the usual asymptotic multiplier ideal sheaves.

Lemma 5.4. Let $\pi: X \longrightarrow Z$ be a projective morphism to a normal affine variety $Z$ and let $D$ be a $\mathbb{Q}$-Cartier divisor. Suppose that $(X, \Delta)$ is log smooth, $\Delta$ is reduced and the stable base locus of $D$ contains no non-Kawamata log terminal centre of $(X, \Delta)$. Then

(1) for any real numbers $0<c_{1} \leq c_{2}$ there is a natural inclusion

$$
\mathcal{J}_{\Delta, c_{2} \cdot\|D\|} \subset \mathcal{J}_{\Delta, c_{1} \cdot\|D\|},
$$

and

(2) if $D$ is Cartier and $S$ is a component of $\Delta$, then the image of the map

$$
\pi_{*} \mathcal{O}_{X}(D) \longrightarrow \pi_{*} \mathcal{O}_{S}(D)
$$

is contained in $\pi_{*} \mathcal{J}_{\Theta,\|D\|_{S}}(D)$, where $\Theta=\left.(\Delta-S)\right|_{S}$.

Proof. (1) is immediate from the definitions.

Suppose that $D$ is Cartier. Pick an integer $p$ such that

$$
\mathcal{J}_{\Theta,\|D\|_{S}}=\mathcal{J}_{\Theta, \frac{1}{p} \cdot|p D|_{S}}
$$

and a $\log$ resolution $\mu: Y \longrightarrow X$ of $|D|,|p D|$ and $(X, \Delta)$. Let $T$ be the strict transform of $S$, let $F_{1}$ be the fixed locus of $\mu^{*}|D|$ and let $F_{p}$ be the fixed locus of $\mu^{*}|p D|$. We have

$$
(\pi \circ \mu)_{*} \mathcal{O}_{Y}\left(\mu^{*} D-F_{1}\right)=\pi_{*} \mathcal{O}_{X}(D)=(\pi \circ \mu)_{*} \mathcal{O}_{Y}\left(E+\mu^{*} D\right) .
$$


The first equality follows by definition of $F_{1}$, and the second follows as $E \geq 0$ is exceptional. As there are inequalities

$$
\mu^{*} D-F_{1} \leq \mu^{*} D-\left\llcorner F_{p} / p\right\lrcorner \leq E+\mu^{*} D-\left\llcorner F_{p} / p\right\lrcorner \leq E+\mu^{*} D,
$$

the image of $\pi_{*} \mathcal{O}_{X}(D)$ is equal to the image of

$$
(\pi \circ \mu)_{*} \mathcal{O}_{Y}\left(E+\mu^{*} D-\left\llcorner F_{p} / p\right\lrcorner\right) .
$$

Thus the image of $\pi_{*} \mathcal{O}_{X}(D)$ is contained in

$$
(\pi \circ \mu)_{*} \mathcal{O}_{T}\left(E+\mu^{*} D-\left\llcorner F_{p} / p\right\lrcorner\right)=\pi_{*} \mathcal{J}_{\Theta,\|D\|_{S}}(D) .
$$

Lemma 5.5. Let $\pi: X \longrightarrow Z$ be a projective morphism to a normal affine variety $Z$ and let $D$ be a Cartier divisor. Suppose that $(X, \Delta)$ is $\log$ smooth and $\Delta$ is reduced. Let $S$ be a component of $\Delta$ and $\Theta=\left.(\Delta-S)\right|_{S}$.

If $\mathbf{B}_{+}(D)$ contains no non-Kawamata log terminal centres of $(X, \Delta)$, then the image of the map

$$
\pi_{*} \mathcal{O}_{X}\left(K_{X}+\Delta+D\right) \longrightarrow \pi_{*} \mathcal{O}_{S}\left(K_{S}+\Theta+D\right)
$$

contains

$$
\pi_{*} \mathcal{J}_{\Theta,\|D\|_{S}}\left(K_{S}+\Theta+D\right) .
$$

Proof. Pick an integer $p>1$ such that

$$
\mathcal{J}_{\Theta,\|D\|_{S}}=\mathcal{J}_{\Theta, \frac{1}{p} \cdot|p D|_{S}},
$$

and there is a divisor $A+B \in|p D|$, where $A \geq 0$ is a general very ample divisor and $B \geq 0$ contains no non-Kawamata $\log$ terminal centres of $(X, \Delta)$. By the resolution lemma of [15, we may find a $\log$ resolution $\mu: Y \longrightarrow X$ of $|p D|$ and of $(X, \Delta)$ which is an isomorphism over every non-Kawamata log terminal centre of $(X, \Delta)$. Let $F_{p}$ be the fixed divisor of $\mu^{*}|p D|, M_{p}=p \mu^{*} D-F_{p}$ and let $\Gamma$ and $T$ be the strict transforms of $\Delta$ and $S$. We have a short exact sequence

$$
0 \longrightarrow \mathcal{O}_{Y}(G-T) \longrightarrow \mathcal{O}_{Y}(G) \longrightarrow \mathcal{O}_{T}(G) \longrightarrow 0
$$

where $G=K_{Y}+\Gamma+\mu^{*} D-\left\llcorner F_{p} / p\right\lrcorner$. As $\mu^{*} A$ is base point free and $\mu^{*}(A+B) \in$ $\mu^{*}|p D|$, the divisor $C=\mu^{*} B-F_{p} \geq 0$. Note that $M_{p}-C \sim \mu^{*} A$. As no component of $C$ is a component of $\Gamma$, we may pick $0<\delta \leq 1 / p$ and an exceptional $\mathbb{Q}$-divisor $F \geq 0$ such that $\left(Y, \Gamma-T+\left\{F_{p} / p\right\}+\delta(C+F)\right)$ is divisorially log terminal and $\mu^{*} A-F$ is ample. As $\left|M_{p}\right|$ is free, $M_{p} / p$ is nef and so

$$
\begin{aligned}
G-T-\left(K_{Y}+\Gamma-T+\left\{\frac{1}{p} F_{p}\right\}+\delta(C+F)\right) & =\frac{1}{p} M_{p}-\delta(C+F) \\
& \sim_{\mathbb{Q}}\left(\frac{1}{p}-\delta\right) M_{p}+\delta\left(\mu^{*} A-F\right)
\end{aligned}
$$

is ample. In particular Kawamata-Viehweg vanishing implies that $R^{1} \phi_{*} \mathcal{O}_{Y}(G-$ $T)=0$, where $\phi=\pi \circ \mu$. Therefore the homomorphism

$$
\pi_{*} \mathcal{O}_{X}\left(K_{X}+\Delta+D\right) \supset \phi_{*} \mathcal{O}_{Y}(G) \longrightarrow \phi_{*} \mathcal{O}_{T}(G)=\pi_{*} \mathcal{J}_{\Theta,\|D\|_{S}}\left(K_{S}+\Theta+D\right)
$$

is surjective. 
Theorem 5.6. Let $\pi: X \longrightarrow Z$ be a projective morphism, where $Z$ is affine and $X$ is a smooth variety of dimension $n$.

If $D$ is a Cartier divisor whose stable base locus is a proper subset of $X, A$ is an ample Cartier divisor and $H$ is a very ample divisor, then $\mathcal{J}_{\|D\|}\left(D+K_{X}+A+n H\right)$ is globally generated.

Proof. Pick an integer $p>0$ such that if $p B \in|p D|$ is a general element, then

$$
\mathcal{J}_{\|D\|}=\mathcal{J}_{\frac{1}{p} \cdot|p D|}=\mathcal{J}_{B} .
$$

Then by (2) of Lemma 4.4, $H^{i}\left(X, \mathcal{J}_{\|D\|}\left(D+K_{X}+A+m H\right)\right)=0$ for all $i>0$ and $m \geq 0$ and we may apply Lemma 5.7 .

The following result is well known to experts. We include a proof for the benefit of the reader:

Lemma 5.7. Let $\pi: X \longrightarrow Z$ be a projective morphism, where $X$ is smooth of dimension $n, Z$ is affine and let $H$ be a very ample divisor.

If $\mathcal{F}$ is any coherent sheaf such that $H^{i}(X, \mathcal{F}(m H))=0$, for $i>0$ and for all $m \geq-n$, then $\mathcal{F}$ is globally generated.

Proof. Pick $x \in X$. Let $\mathcal{T} \subset \mathcal{F}$ be the torsion subsheaf supported at $x$, and let $\mathcal{G}=\mathcal{F} / \mathcal{T}$. Then $H^{i}(X, \mathcal{G}(m H))=0$ for $i>0$ and for all $m \geq-n$ and $\mathcal{F}$ is globally generated if and only if $\mathcal{G}$ is globally generated. Replacing $\mathcal{F}$ by $\mathcal{G}$ we may therefore assume that $\mathcal{T}=0$.

Pick a general element $Y \in|H|$ containing $x$. As $\mathcal{T}=0$ there is an exact sequence

$$
0 \longrightarrow \mathcal{F}(-Y) \longrightarrow \mathcal{F} \longrightarrow \mathcal{G} \longrightarrow 0
$$

where $\mathcal{G}=\mathcal{F} \otimes \mathcal{O}_{Y}$. As $H^{i}(Y, \mathcal{G}(m H))=0$, for $i>0$ and for all $m \geq-(n-1)$, $\mathcal{G}$ is globally generated by induction on the dimension. As $H^{1}(X, \mathcal{F}(-Y))=0$ it follows that $\mathcal{F}$ is globally generated.

Proof of Theorem [5.3. We follow the argument of [5], which in turn is based on the ideas of [7, [14] and [17.

We proceed by induction on $m$. The statement is clear for $m=0$, and so it suffices to show that

$$
\mathcal{J}_{\left\|\left.(m+1) D\right|_{S}\right\|} \subset \mathcal{J}_{\Theta,\|(m+1) D+P\|_{S}}
$$

assuming that

$$
\mathcal{J}_{\left\|\left.t D\right|_{S}\right\|} \subset \mathcal{J}_{\Theta,\|t D+P\|_{S}} \quad \text { for all } \quad t \leq m .
$$

If $\Delta=\sum \delta_{i} \Delta_{i}$, where each $\Delta_{i}$ is a prime divisor, then for any $1 \leq s \leq k$, put

$$
\Delta^{s}=\sum_{i: \delta_{i}>(k-s) / k} \Delta_{i}
$$

We have

- each $\Delta^{s}$ is integral,

- $S=\Delta^{1} \leq \Delta^{2} \leq \cdots \leq \Delta^{k}=\ulcorner\Delta\urcorner$, and

- $\Delta=\frac{1}{k} \sum_{s=1}^{k} \Delta^{s}$, 
and these properties uniquely determine the divisors $\Delta^{s}$. We let $\Delta^{k+1}=\ulcorner\Delta\urcorner$. We recursively define integral divisors $D_{\leq s}$ by the rule

$$
D_{\leq s}= \begin{cases}0 & \text { if } s=0, \\ K_{X}+\Delta^{s}+D_{\leq s-1} & 1 \leq s \leq k .\end{cases}
$$

Note that $D_{\leq k}=D$. By (1) of Lemma 5.4 there is an inclusion

$$
\mathcal{J}_{\left\|\left.(m+1) D\right|_{S}\right\|} \subset \mathcal{J}_{\left\|\left.m D\right|_{S}\right\|}
$$

and so it suffices to prove that there are inclusions

$$
\mathcal{J}_{\left\|\left.m D\right|_{S}\right\|} \subset \mathcal{J}_{\Theta^{s+1},\left\|m D+D_{\leq s}+P\right\|_{S}},
$$

for $0 \leq s \leq k$, where $\Theta^{i}=\left.\left(\Delta^{i}-S\right)\right|_{S}$ for $1 \leq i \leq k+1$. Thus $\Theta^{k}=\Theta^{k+1}=\Theta$ and $\Theta^{1}=0$.

We proceed by induction on $s$. Now

$$
\mathcal{J}_{\left\|\left.m D\right|_{S}\right\|} \subset \mathcal{J}_{\Theta,\|m D+P\|_{S}} \subset \mathcal{J}_{\Theta^{1},\|m D+P\|_{S}}
$$

The first inclusion holds by assumption and since $\Theta^{1} \leq \Theta$, (2) of Lemma 4.3 implies the second inclusion. Thus (因) holds when $s=0$.

Now suppose that (因) holds for $s \leq t-1$. Note that

$$
\begin{aligned}
m D+D_{\leq t}+P & =K_{X}+\Delta^{t}+\left(D_{\leq t-1}+P\right)+m D \\
& =m D+K_{X}+\left(\Delta^{t}+D_{\leq t-1}+r A\right)+n Q
\end{aligned}
$$

where, by assumption, both $D_{\leq t-1}+P$ and $\Delta^{t}+D_{\leq t-1}+r A$ are ample for any $1 \leq t \leq k+1$. In particular $\mathbf{B}_{+}\left(m D+D_{\leq t-1}+P\right)$ contains no non-Kawamata log terminal centres of $(X,\ulcorner\Delta\urcorner)$. Then

$$
\begin{gathered}
\pi_{*} \mathcal{J}_{\left\|\left.m D\right|_{S}\right\|}\left(m D+D_{\leq t}+P\right) \subset \pi_{*} \mathcal{J}_{\Theta^{t},\left\|m D+D_{\leq t-1}+P\right\|_{S}}\left(m D+D_{\leq t}+P\right) \\
\subset \operatorname{Im}\left(\pi_{*} \mathcal{O}_{X}\left(m D+D_{\leq t}+P\right) \longrightarrow \pi_{*} \mathcal{O}_{S}\left(m D+D_{\leq t}+P\right)\right) \\
\subset \pi_{*} \mathcal{J}_{\Theta^{t+1},\left\|m D+D_{\leq t}+P\right\|_{S}}\left(m D+D_{\leq t}+P\right) .
\end{gathered}
$$

The first inclusion holds as we are assuming (因) for $s=t-1$, the second inclusion holds by (†) and Lemma 5.5 and the last inclusion follows from (2) of Lemma 5.4. But (†) and Theorem 5.6 imply that

$$
\mathcal{J}_{\left\|\left.m D\right|_{S}\right\|}\left(m D+D_{\leq t}+P\right)
$$

is generated by global sections and so

$$
\mathcal{J}_{\left\|\left.m D\right|_{S}\right\|} \subset \mathcal{J}_{\Theta^{t+1},\left\|m D+D_{\leq t}+P\right\|_{S}}
$$

The inclusion

$$
\pi_{*} \mathcal{J}_{\left\|\left.m D\right|_{S}\right\|}(m D+P) \subset \operatorname{Im}\left(\pi_{*} \mathcal{O}_{X}(m D+P) \rightarrow \pi_{*} \mathcal{O}_{S}(m D+P)\right)
$$

is part of the inclusions proved above when $s=k$. 


\section{LifTING SECTIONS}

Lemma 6.1. Let $D \geq 0$ be a Cartier divisor on a normal variety $X$, and let $Z \subset X$ be an irreducible subvariety.

Then

$$
\liminf \frac{\operatorname{mult}_{Z}(|m D|)}{m}=\lim \frac{\operatorname{mult}_{Z}(|m ! D|)}{m !} .
$$

Proof. Note that if $a$ divides $b$, then

$$
\frac{\text { mult }_{Z}(|a D|)}{a} \geq \frac{\operatorname{mult}_{Z}(|b D|)}{b},
$$

whence the result.

The following is essentially proved in [4; we include a proof for the benefit of the reader:

Lemma 6.2. Let $D \subset X$ be a divisor on a smooth variety and $Z$ a closed subvariety. If $\lim \operatorname{mult}_{Z}(|m ! D|) / m !=0$, then $Z$ is not contained in $\mathbf{B}_{-}(D)$.

Proof. Let $A$ be any ample divisor. Pick $l>0$ such that $l A-K_{X}$ is ample. If $m>l$ is sufficiently divisible, then $\mathcal{J}_{\|m D\|}(m(D+A))$ is globally generated by Theorem 5.6. But if $p>0$ is sufficiently large and divisible and $D_{m p} \in|m p D|$ is general, then mult $_{Z} D_{m p}=$ mult $_{Z}|m p D|<p$ and

$$
\mathcal{J}_{\|m D\|}=\mathcal{J}_{(1 / p) D_{m p}} \text {. }
$$

But since mult ${ }_{Z} D_{m p} / p<1$ it follows that $\left(X, D_{m p} / p\right)$ is Kawamata log terminal, in a neighbourhood of the generic point of $Z$. Thus $Z$ is not contained in the co-support of $\mathcal{J}_{\|m D\|}$ and so $Z$ is not contained in the base locus of $m(D+A)$.

Theorem 6.3. Let $\pi: X \longrightarrow Z$ be a projective morphism to a normal affine variety $Z$, where $(X, \Delta=S+A+B)$ is a purely log terminal pair, $S=\llcorner\Delta\lrcorner$ is irreducible, $(X, S)$ is log smooth, $A \geq 0$ is a general ample $\mathbb{Q}$-divisor, $B \geq 0$ is a $\mathbb{Q}$-divisor and $\left(S, \Omega+\left.A\right|_{S}\right)$ is canonical, where $\Omega=\left.(\Delta-S)\right|_{S}$. Assume that the stable base locus of $K_{X}+\Delta$ does not contain $S$. Let $F=\lim F_{l !}$, where, for any positive and sufficiently divisible integer $m$, we let

$$
F_{m}=\operatorname{Fix}\left(\left|m\left(K_{X}+\Delta\right)\right|_{S}\right) / m .
$$

If $\epsilon>0$ is any rational number such that $\epsilon\left(K_{X}+\Delta\right)+A$ is ample and if $\Phi$ is any $\mathbb{Q}$-divisor on $S$ and $k>0$ is any integer such that

(1) both $k \Delta$ and $k \Phi$ are Cartier, and

(2) $\Omega \wedge \lambda F \leq \Phi \leq \Omega$, where $\lambda=1-\epsilon / k$, then

$$
\left|k\left(K_{S}+\Omega-\Phi\right)\right|+k \Phi \subset\left|k\left(K_{X}+\Delta\right)\right|_{S} .
$$

Proof. By assumption $A=H / m$, where $H$ is very ample and a very general element of $|H|$ and $m \geq 2$ is an integer. If $C=A / k$, then

$$
A+(k-1) C=\frac{2 k-1}{k m} H,
$$

and so

$$
\left(X, \Delta+(k-1) C=S+\frac{2 k-1}{k m} H+B\right)
$$


is purely log terminal, as

On the other hand,

$$
\frac{2 k-1}{k m}<1
$$

$$
\left(S, \Omega+\left.C\right|_{S}\right)
$$

is canonical as we are even assuming that $\left(S, \Omega+\left.A\right|_{S}\right)$ is canonical. Pick $\eta>\epsilon / k$ rational so that $\eta\left(K_{X}+\Delta\right)+C$ is ample and let $\mu=1-\eta<\lambda=1-\epsilon / k$. If $l>0$ is any sufficiently divisible integer so that $O=l\left(\eta\left(K_{X}+\Delta\right)+C\right)$ is very ample, then

$$
\begin{aligned}
G_{l} & =\operatorname{Fix}\left(\left|l\left(K_{X}+\Delta+C\right)\right|_{S}\right) / l \\
& =\operatorname{Fix}\left(\left|l \mu\left(K_{X}+\Delta\right)+O\right|_{S}\right) / l \\
& \leq \operatorname{Fix}\left(\left|l \mu\left(K_{X}+\Delta\right)\right|_{S}\right) / l \\
& =\mu F_{\mu l} .
\end{aligned}
$$

Thus

$$
\lim G_{l !} \leq \mu \lim F_{l !}=\mu F \text {. }
$$

On the other hand, Lemma 6.2 implies that there is a positive integer $l$ such that every prime divisor on $S$ which does not belong to the support of $F$ does not belong to the base locus of $\left|l\left(K_{X}+\Delta+C\right)\right|$. Thus we may pick a positive integer $l$ such that

- $k$ divides $l$,

- $l C$ is Cartier, and

- $G_{l} \leq \lambda F$.

Let $f: Y \longrightarrow X$ be a $\log$ resolution of the linear system $\left|l\left(K_{X}+\Delta+C\right)\right|$ and of $(X, \Delta+C)$. We may write

$$
K_{Y}+\Gamma=f^{*}\left(K_{X}+\Delta+C\right)+E,
$$

where $\Gamma \geq 0$ and $E \geq 0$ have no common components, $f_{*} \Gamma=\Delta+C$ and $f_{*} E=0$. Then

$$
H_{l}=\operatorname{Fix}\left(l\left(K_{Y}+\Gamma\right)\right) / l=\operatorname{Fix}\left(l f^{*}\left(K_{X}+\Delta+C\right)\right) / l+E .
$$

If $\Xi=\Gamma-\Gamma \wedge H_{l}$, then $l\left(K_{Y}+\Xi\right)$ is Cartier and $\operatorname{Fix}\left(l\left(K_{Y}+\Xi\right)\right)$ and $\Xi$ share no common components. Since the mobile part of $\left|l\left(K_{Y}+\Xi\right)\right|$ is free and the support of $\operatorname{Fix}\left(l\left(K_{Y}+\Xi\right)\right)+\Xi$ has normal crossings it follows that the stable base locus of $K_{Y}+\Xi$ contains no non-Kawamata $\log$ terminal centres of $(Y,\ulcorner\Xi\urcorner$ ) (which are nothing but the strata of $\ulcorner\Xi\urcorner$ ).

Let $H \geq 0$ be any ample divisor on $Y$. Pick positive integers $m$ and $q$ such that $l$ divides $m$ and $Q=q H$ is very ample. Let $T$ be the strict transform of $S$, let $\Gamma_{T}=\left.(\Gamma-T)\right|_{T}$ and let $\Xi_{T}=\left.(\Xi-T)\right|_{T}$. If

$$
\tau \in H^{0}\left(T, \mathcal{O}_{T}\left(m\left(K_{T}+\Xi_{T}\right)\right)\right)=H^{0}\left(T, \mathcal{J}_{\left\|m\left(K_{T}+\Xi_{T}\right)\right\|}\left(m\left(K_{T}+\Xi_{T}\right)\right)\right),
$$

and $\sigma \in H^{0}\left(T, \mathcal{O}_{T}(Q)\right)$, then

$$
\sigma \cdot \tau \in H^{0}\left(T, \mathcal{J}_{\left\|m\left(K_{T}+\Xi_{T}\right)\right\|}\left(m\left(K_{T}+\Xi_{T}\right)+Q\right)\right) .
$$

On the other hand, if $q$ is sufficiently large and divisible, then by Theorem 5.3 $H^{0}\left(T, \mathcal{J}_{\left\|m\left(K_{T}+\Xi_{T}\right)\right\|}\left(m\left(K_{T}+\Xi_{T}\right)+Q\right)\right)$ is contained in the image of

$$
H^{0}\left(Y, \mathcal{O}_{Y}\left(m\left(K_{Y}+\Xi\right)+Q\right)\right) \longrightarrow H^{0}\left(T, \mathcal{O}_{T}\left(m\left(K_{T}+\Xi_{T}\right)+Q\right)\right) .
$$


Hence there is a fixed $q$ such that whenever $l$ divides $m$, we have

$$
\left|m\left(K_{T}+\Xi_{T}\right)\right|+m\left(\Gamma_{T}-\Xi_{T}\right)+|Q|_{T}|\subset| m\left(K_{Y}+\Gamma\right)+\left.Q\right|_{T} .
$$

If $g=\left.f\right|_{T}: T \longrightarrow S$, then $g_{*} \Gamma_{T}=\Omega+\left.C\right|_{S}$ and since $g_{*} \Xi_{T} \leq \Omega+\left.C\right|_{S}$ and $\left(S, \Omega+\left.C\right|_{S}\right)$ is canonical, we have $\left|m\left(K_{S}+g_{*} \Xi_{T}\right)\right|=g_{*}\left|m\left(K_{T}+\Xi_{T}\right)\right|$. Therefore, applying $g_{*}$, we obtain

$$
\left|m\left(K_{S}+g_{*} \Xi_{T}\right)\right|+m\left(\Omega+\left.C\right|_{S}-g_{*} \Xi_{T}\right)+\left.P\right|_{S} \subset\left|m\left(K_{X}+\Delta+C\right)+P\right|_{S},
$$

where $P=f_{*} Q$.

Since for every prime divisor $L$ on $S$ we have

$$
\operatorname{mult}_{L} G_{l}=\operatorname{mult}_{L^{\prime}} \operatorname{Fix}\left(\left|l\left(K_{Y}+\Gamma\right)\right|_{T}\right) / l=\text { mult }\left._{L^{\prime}} H_{l}\right|_{T},
$$

where $L^{\prime}$ is the strict transform of $L$ on $T$, it follows that

$$
g_{*} \Xi_{T}-\left.C\right|_{S}=\Omega-\Omega \wedge G_{l} \geq \Omega-\Omega \wedge \lambda F \geq \Omega-\Phi \geq 0 .
$$

Therefore

$$
\left|m\left(K_{S}+\Omega-\Phi\right)\right|+m \Phi+\left.(m C+P)\right|_{S} \subset\left|m\left(K_{X}+\Delta+C\right)+P\right|_{S},
$$

for any $m$ divisible by $l$. In particular, if we pick $m$ so that $C-\frac{k-1}{m} P$ is ample and $\left(X, \Delta+\frac{k-1}{m} P\right)$ is purely log terminal, then the result follows by Theorem 4.1.

\section{Rationality of The ReStricted AlgeBRA}

In this section we will prove:

Theorem 7.1. Assume Theorem $\mathrm{F}_{-1}$.

Let $\pi: X \longrightarrow Z$ be a projective morphism to a normal affine variety $Z$, where $(X, \Delta=S+A+B)$ is a purely log terminal pair of dimension $n, S=\llcorner\Delta\lrcorner$ is irreducible, $(X, S)$ is log smooth, $A \geq 0$ is a general ample $\mathbb{Q}$-divisor, $B \geq 0$ is a $\mathbb{Q}$-divisor and $\left(S, \Omega+\left.A\right|_{S}\right)$ is canonical, where $\Omega=\left.(\Delta-S)\right|_{S}$. Assume that the stable base locus of $K_{X}+\Delta$ does not contain $S$. Let $F=\lim F_{l !}$, where, for any positive and sufficiently divisible integer $m$, we let

$$
F_{m}=\operatorname{Fix}\left(\left|m\left(K_{X}+\Delta\right)\right|_{S}\right) / m .
$$

Then $\Theta=\Omega-\Omega \wedge F$ is rational. Moreover if both $k \Delta$ and $k \Theta$ are Cartier, then

$$
\left|k\left(K_{S}+\Theta\right)\right|+k(\Omega-\Theta)=\left|k\left(K_{X}+\Delta\right)\right|_{S}
$$

and

$$
R_{S}\left(X, k\left(K_{X}+\Delta\right)\right) \simeq R\left(S, k\left(K_{S}+\Theta\right)\right) .
$$

Proof. Suppose that $\Theta$ is not rational. Let $V \subset \operatorname{WDiv}_{\mathbb{R}}(S)$ be the vector space spanned by the components of $\Theta$. Then there is a constant $\delta>0$ such that if $\Phi \in V$ and $\|\Phi-\Theta\|<\delta$, then $\Phi \geq 0$ has the same support as $\Theta$ and moreover, by (2) of Theorem $\mathrm{F}_{-1}$, if $G$ is a prime divisor contained in the stable base locus of $K_{S}+\Theta$, then it is also contained in the stable base locus of $K_{S}+\Phi$.

If $l\left(K_{X}+\Delta\right)$ is Cartier and $\Theta_{l}=\Omega-\Omega \wedge F_{l}$, then

$$
\left|l\left(K_{X}+\Delta\right)\right|_{S} \subset\left|l\left(K_{S}+\Theta_{l}\right)\right|+l\left(\Omega \wedge F_{l}\right) .
$$

Hence $\operatorname{Fix}\left(l\left(K_{S}+\Theta_{l}\right)\right)$ does not contain any components of $\Theta_{l}$. In particular the stable base locus of $K_{S}+\Theta_{l}$ does not contain any components of $\Theta_{l}$. But we may pick $l>0$ so that $\Theta_{l} \in V$ and $\left\|\Theta_{l}-\Theta\right\|<\delta$. It follows that no component of $\Theta$ is in the stable base locus of $K_{S}+\Theta$. 
Let $W \subset V$ be the smallest rational affine space which contains $\Theta$. (3) of Theorem $\mathrm{F}_{h-1}$ implies that there is a positive integer $r>0$ and a positive constant $\eta>0$ such that if $\Phi \in W, k \Phi / r$ is Cartier and $\|\Phi-\Theta\|<\eta$, then every component of $\operatorname{Fix}\left(k\left(K_{S}+\Phi\right)\right)$ is in fact a component of the stable base locus of $K_{S}+\Theta$.

Pick a rational number $\epsilon>0$ such that $\epsilon\left(K_{X}+\Delta\right)+A$ is ample. By Diophantine approximation, see (3.7.7) of 2], we may find a positive integer $k$, a divisor $\Phi$ on $S$ and a prime divisor $G$ (necessarily a component of $\Theta$ whose coefficient is irrational) such that

(1) $0 \leq \Phi \in W$,

(2) both $k \Phi / r$ and $k \Delta / r$ are Cartier,

(3) $\|\Phi-\Theta\|<\min (\delta, \eta, f \epsilon / k)$, where $f$ is the smallest non-zero coefficient of $F \neq 0$, and

(4) $\operatorname{mult}_{G} \Phi>\operatorname{mult}_{G} \Theta$.

Claim 7.2. $\Omega \wedge \lambda F \leq \Omega-\Phi \leq \Omega$, where $\lambda=1-\epsilon / k$.

Proof of Claim 7.2. Let $P$ be a prime divisor on $S$ and let $\omega, f, \phi$ and $\theta$ be the multiplicities of $\Omega, F, \Phi$ and $\Theta$ along $P$. We just need to check that

$$
\min (\omega, \lambda f) \leq \omega-\phi .
$$

There are two cases. If $\omega \leq f$, then $\theta=0$ so that $\phi=0$ and (因) holds. If $\omega \geq f$, then $\theta=\omega-f$ and since $\|\Phi-\Theta\|<f \epsilon / k$,

$$
\min (\omega, \lambda f)=\left(1-\frac{\epsilon}{k}\right) f \leq f-(\phi-\theta)=\omega-\phi
$$

Claim 7.2 (2) and Theorem 6.3 imply that

$$
\left|k\left(K_{S}+\Phi\right)\right|+k(\Omega-\Phi) \subset\left|k\left(K_{X}+\Delta\right)\right|_{S}
$$

(4) implies that $G$ is a component of $\operatorname{Fix}\left(k\left(K_{S}+\Phi\right)\right)$. (2) and $\|\Phi-\Theta\|<\eta$ imply that $G$ is a component of the stable base locus of $K_{S}+\Theta$, a contradiction.

Thus $\Theta$ is rational. Hence $\Omega \wedge F$ is rational, and we are done by Theorem 6.3

\section{Proof of Theorem 1.3}

Theorem 8.1. Assume Theorem $\mathrm{F}_{h-1}$.

Let $\pi: X \longrightarrow Z$ be a projective morphism to a normal affine variety $Z$. Suppose that $(X, \Delta=S+A+B)$ is a purely log terminal pair of dimension $n, S=\llcorner\Delta\lrcorner$ is irreducible and not contained in the stable base locus of $K_{X}+\Delta, A \geq 0$ is a general ample $\mathbb{Q}$-divisor and $B \geq 0$ is a $\mathbb{Q}$-divisor.

Then there is a birational morphism $g: T \longrightarrow S$, a positive integer $l$ and a Kawamata log terminal pair $(T, \Theta)$ such that $K_{T}+\Theta$ is $\mathbb{Q}$-Cartier and

$$
R_{S}\left(X, l\left(K_{X}+\Delta\right)\right) \cong R\left(T, l\left(K_{T}+\Theta\right)\right) .
$$

Proof. If $f: Y \longrightarrow X$ is a $\log$ resolution of $(X, \Delta)$, then we may write

$$
K_{Y}+\Gamma^{\prime}=f^{*}\left(K_{X}+\Delta\right)+E,
$$

where $\Gamma^{\prime} \geq 0$ and $E \geq 0$ have no common components, $f_{*} \Gamma^{\prime}=\Delta$ and $f_{*} E=0$. If $T$ is the strict transform of $S$, then we may choose $f$ so that $\left(T, \Psi^{\prime}=\left.\left(\Gamma^{\prime}-T\right)\right|_{T}\right)$ is terminal. Note that $T$ is not contained in the stable base locus of $K_{Y}+\Gamma^{\prime}$ as $S$ is not contained in the stable base locus of $K_{X}+\Delta$. 
Pick a $\mathbb{Q}$-divisor $F$ such that $f^{*} A-F$ is ample and $\left(Y, \Gamma^{\prime}+F\right)$ is purely log terminal. Pick $m>1$ so that $m\left(f^{*} A-F\right)$ is very ample and pick $m C \in\left|m\left(f^{*} A-F\right)\right|$ very general. Then

$$
\left(Y, \Gamma=\Gamma^{\prime}-f^{*} A+F+C \sim_{\mathbb{Q}} \Gamma^{\prime}\right)
$$

is purely $\log$ terminal (note that since $A$ is general, $f^{*} A$ is equal to the strict transform of $A)$ and if $m$ is sufficiently large, $\left(T, \Psi+\left.C\right|_{T}\right)$ is terminal, where $\Psi=\left.(\Gamma-T)\right|_{T}$.

On the other hand,

$$
\begin{aligned}
R\left(X, k\left(K_{X}+\Delta\right)\right) & \cong R\left(Y, k\left(K_{Y}+\Gamma\right)\right) \quad \text { and } \\
R_{S}\left(X, k\left(K_{X}+\Delta\right)\right) & \cong R_{T}\left(Y, k\left(K_{Y}+\Gamma\right)\right),
\end{aligned}
$$

for any $k$ sufficiently divisible. Now apply Theorem 7.1 to $(Y, \Gamma)$.

Proof of Theorem [1.3. By Theorem 3.2 we may assume that $Z$ is affine and by Lemma 3.5, it suffices to prove that the restricted algebra is finitely generated. As $Z$ is affine, $S$ is mobile and as $f$ is birational, the divisor $\Delta-S$ is big. But then

$$
\Delta-S \sim_{\mathbb{Q}} A+B,
$$

where $A$ is a general ample $\mathbb{Q}$-divisor and $B \geq 0$. As $S$ is mobile, we may assume that the support of $B$ does not contain $S$. Now

$$
K_{X}+\Delta^{\prime}=K_{X}+S+(1-\epsilon)(\Delta-S)+\epsilon A+\epsilon B \sim_{\mathbb{Q}} K_{X}+\Delta
$$

is purely $\log$ terminal, where $\epsilon$ is any sufficiently small positive rational number. By Lemma 3.4 we may replace $\Delta$ by $\Delta^{\prime}$. We may therefore assume that $\Delta=$ $S+A+B$, where $A$ is a general ample $\mathbb{Q}$-divisor and $B \geq 0$. Since we are assuming Theorem $\mathrm{F}_{h-1}$, Theorem 8.1 implies that the restricted algebra is finitely generated.

\section{REFERENCES}

1. F. Ambro, Restrictions of log canonical algebras of general type, J. Math. Sci. Univ. Tokyo 13 (2006), no. 3, 409-437. MR2284409 (2007m:14015)

2. C. Birkar, P. Cascini, C. Hacon, and J. M Kernan, Existence of minimal models for varieties of log general type. J. Amer. Math. Soc., posted on November 13, 2009, PII: S 08940347(09)00649-3.

3. A. Corti, 3-fold flips after Shokurov, Flips for 3-folds and 4-folds (Alessio Corti, ed.), Oxford University Press, 2007, pp. 18-48. MR2359340

4. L. Ein, R. Lazarsfeld, M. Mustaţă, M. Nakamaye, and M. Popa, Asymptotic invariants of base loci, Ann. Inst. Fourier (Grenoble) 56 (2006), no. 6, 1701-1734. MR2282673 (2007m:14008)

5. C. Hacon and J. $\mathrm{M}^{\mathrm{C}}$ Kernan, Boundedness of pluricanonical maps of varieties of general type, Invent. Math. 166 (2006), no. 1, 1-25. MR2242631 (2007e:14022)

6. Extension theorems and the existence of flips, Flips for 3-folds and 4-folds (Alessio Corti, ed.), Oxford University Press, 2007, pp. 79-100. MR2352762 (2008j:14031)

7. Y. Kawamata, On the extension problem of pluricanonical forms, Algebraic geometry: Hirzebruch 70 (Warsaw, 1998), Contemp. Math., vol. 241, Amer. Math. Soc., Providence, RI, 1999, pp. 193-207. MR.1718145 (2000i:14053)

8. J. Kollár and S. Mori, Birational geometry of algebraic varieties, Cambridge Tracts in Mathematics, vol. 134, Cambridge University Press, 1998. MR.1658959 (2000b:14018)

9. R. Lazarsfeld, Positivity in algebraic geometry. I, Ergebnisse der Mathematik und ihrer Grenzgebiete. 3. Folge. A Series of Modern Surveys in Mathematics [Results in Mathematics and Related Areas. 3rd Series. A Series of Modern Surveys in Mathematics], vol. 48, Springer-Verlag, Berlin, 2004, Classical setting: Line bundles and linear series. MR2095471 (2005k:14001a) 
10. - Positivity in algebraic geometry. II, Ergebnisse der Mathematik und ihrer Grenzgebiete. 3. Folge. A Series of Modern Surveys in Mathematics [Results in Mathematics and Related Areas. 3rd Series. A Series of Modern Surveys in Mathematics], vol. 49, Springer-Verlag, Berlin, 2004, Positivity for vector bundles, and multiplier ideals. MR2095472 (2005k:14001b)

11. N. Nakayama, Zariski-decomposition and abundance, MSJ Memoirs, vol. 14, Mathematical Society of Japan, Tokyo, 2004. MR2104208 (2005h:14015)

12. V. V. Shokurov, Prelimiting flips, Proc. Steklov Inst. of Math. 240 (2003), 82-219. MR $1993750(2004 \mathrm{k}: 14024)$

13. Y-T. Siu, A General Non-Vanishing Theorem and an Analytic Proof of the Finite Generation of the Canonical Ring. arXiv:math.AG/0610740

14. - Invariance of plurigenera, Invent. Math. 134 (1998), no. 3, 661-673. MR1660941 (99i:32035)

15. E. Szabó, Divisorial log terminal singularities, J. Math. Sci. Univ. Tokyo 1 (1994), no. 3, 631-639. MR1322695 (96f:14019)

16. S. Takayama, Pluricanonical systems on algebraic varieties of general type, Invent. Math. 165 (2006), no. 3, 551-587. MR2242627 (2007m:14014)

17. H. Tsuji, Pluricanonical systems of projective varieties of general type. arXiv:math.AG/ 9909021

Department of Mathematics, University of Utah, 155 South 1400 East, JWB 233, Salt Lake City, Utah 84112

E-mail address: hacon@math.utah.edu

Department of Mathematics, University of California at Santa Barbara, Santa Barbara, California 93106 and Department of Mathematics, mit, 77 Massachusetts Avenue, Cambridge, Massachusetts 02139

E-mail address: mckernan@math.ucsb.edu

E-mail address: mckernan@math.mit.edu 